\title{
Femoral Neck Stress Fracture
}
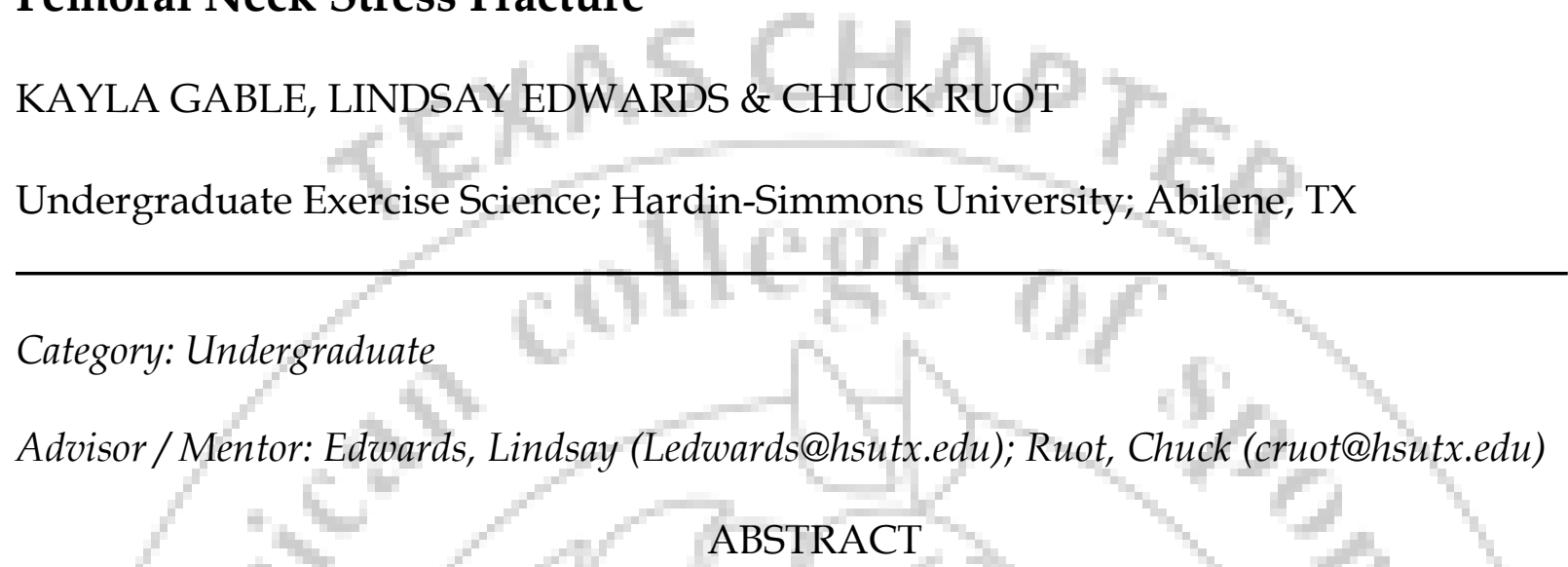

CLINICAL PRESENTATION \& EXAM: A patient with a femoral neck stress fracture will complain of persistent deep groin pain, orientated anteriorly. The patient will note pain as a new sensation in that area of the body that increases during activity. A patient with a femoral neck stress fracture will have tenderness to palpation. Pain from femoral neck stress fractures occur with repeated vigorous training, such as running, rather than trauma. During a physical examination by a medical professional, a patient suffering from a femoral neck stress fracture will have a gait that favors one leg, the fractured leg will present itself shorter than the un-fractured leg, or the fractured leg could be rotated externally with the knee and foot outwardly turned. Upon further examination, via magnetic resonance imaging or X-Ray, a dense line will present itself in the femoral neck indicating a fracture. ANATOMY \& PATHOLOGY: The femur is the longest and most dense bone in the human body, making it an excellent weight-bearing supporter during walking, standing, running and jumping. Since the muscles surrounding the femur are larger muscles, such as the quadriceps, intense forces act on the femur to illicit movement. At the proximal end of the femur is the head of the femur, which is smooth and round forming the ball-and socket joint of the hip which articulates at the acetabulum. Distally from the head of the femur is the femoral neck; the femoral neck is considerably more narrow; this allows the leg to have more range of motion at the hip joint, however the thinness of the femoral neck increases the susceptibility to fractures. To combat the likelihood of a fracture in the femoral neck, the greater and lesser trochanters provide width and strength to the femur. At the acetabulum, the femur aids in the flexion, extension, abduction and adduction of the leg. A femoral neck stress fracture limits internal rotation of the femur. DIAGNOSTIC TESTING \& CONSIDERATIONS: A femoral neck stress fracture can be diagnosed by examining the position of the patient's hip and leg, along with their symptoms. A recommendation of an X-Ray is usually given following an examination by a medical professional. X-Ray's may not be able to detect miniscule hairline or incomplete fractures; therefore, a CT scan, an MRI or a bone scan may be recommended. If a femoral neck stress fracture is not diagnosed within a proper time frame, blood vessels that supply the femoral head could tear; resulting in the femoral head not having adequate blood supply, leading to avascular necrosis and the inevitable collapse of the femur. TREATMENT \& RETURN TO ACTIVITY: Treatment of a femoral neck stress fracture depends on the severity. Femoral neck stress fracture treatments can include surgery and rehabilitation. . Whether there is displacement causing the blood supply to be compromised to the femoral head determines the type of surgery needed. If blood supply is disrupted, partial or total hip replacement is required. Internal fixation involves the use of metal screws or pins that are either implanted into the femur or secured to a metal plate along the femur. This is used when there is no displacement compromising blood supply. Post-surgical physical therapy is needed in order to restore mobility and strength. Medication can be a source of treatment in the early stages, but it only offers shortterm pain relief and does not heal the fracture. A non-surgical treatment may involve spending six to eight weeks non-weightbearing. Return to activity depends on the severity of the fracture and type of treatment. 